\title{
Analysis of Salivary Micronutrients in Oral Potentially Malignant Disorder
}

Sham Kishor Kanneppady ${ }^{1 *}$, Anusha Bhaskar ${ }^{2}$, Sowmya Sham Kanneppady ${ }^{3}$, Prasanna Kumar Rao ${ }^{4}$, Shamala Ramasamy $^{5}$, Anusha Rangare Lakshman ${ }^{6}$, Shishir Ram Shetty ${ }^{7}$, Ullal Harshini Devi ${ }^{8}$, Aung Myo Oo $^{9}$ and Ohn Mar Lwin ${ }^{10}$

${ }^{1}$ Senior Lecturer, Division of Oral Diagnostics and Surgical Sciences, School of Dentistry, International Medical University, Kuala Lumpur, Malaysia

${ }^{2}$ Associate Professor and Senior Scientist, Department of Biochemistry, PRIST University, Thanjavur, Tamil Nadu, India

${ }^{3}$ Senior Lecturer and Head, Department of Pharmacology, Faculty of Medicine, Lincoln University College, Selangor Darul Ehsan, Malaysia

${ }^{4}$ Professor and Head, Department of Oral Medicine and Radiology, A.J. Institute of Dental Sciences, Kuntikana, Mangalore, India

${ }^{5}$ Lecturer and Head of Division of Psychology, School of Medicine, International Medical University, Kuala Lumpur, Malaysia

${ }^{6}$ Reader and Head, Department of Oral Medicine and Radiology, Century International Institute of Dental Sciences and Research Centre, Poinachi, Kasaragod, Kerala, India

${ }^{7}$ Assistant Professor, Department of Oral Medicine and Radiology, Gulf Medical University, UAE

${ }^{8}$ Department of Biotechnology Engineering, NMAMIT, Nitte, Karnataka, India

${ }^{9}$ Assistant Professor, Department of Biochemistry, Faculty of Medicine, Lincoln University College, Selangor Darul Ehsan, Malaysia

${ }^{10}$ Post graduate student, Department of Physiology, Faculty of Medicine, University Malaya, Kuala Lumpur, Malaysia

Received: March 20, 2018; Accepted: May 4, 2018; Published: May 08, 2018

*Corresponding author: Sham Kishor Kanneppady, Senior Lecturer, Division of Oral Diagnostics and Surgical Sciences, School of Dentistry, International Medical University, Kuala Lumpur, Malaysia, Fax: +60386567229, E-mail: drsham.omr@gmail.com

\section{Abstract}

Background: Sound nutritional habits and a sufficient supply of essential vitamins and minerals are of considerable importance for oral health This study was aimed to evaluate whether salivary micronutrients play any role in diagnosis or prognostic estimation of Oral Sub mucous Fibrosis (OSF), one of the oral potentially malignant disorders.
\end{abstract}

Materials and methods: In this case control study, 40 histopathologically confirmed cases of OSF were compared with 44 age and sex matched healthy controls. Salivary samples collected from the participants were centrifuged at $2500 \mathrm{rpm}$ for 10 minutes. The supernatant collected was stored at $-20^{\circ} \mathrm{C}$. The saliva was then analysed for micronutrients. Zinc and $\mathrm{Cu}$ level were analysed using atomic absorption spectrometer and Fe using spectrophotometer.

Results: The mean age of patients with OSF was 38.28 2.23 years. Among them, 31 (77.5\%) were males and $9(22.5 \%)$ were females. The mean salivary $\mathrm{Cu}$ values of OSF group $(127.30 \pm 6.77 \mu \mathrm{g} / \mathrm{dl})$ has increased compared to that of controls $(112.68 \pm 7.95 \mu \mathrm{g} / \mathrm{dl})$. The mean salivary $\mathrm{Zn}$ values of OSF group $(116.80 \pm 9.93 \mu \mathrm{g} / \mathrm{dl})$ was significantly decreased $(P<0.01)$ compared to that of controls $(146.76 \pm 9.17 \mu \mathrm{g} / \mathrm{dl})$. The mean salivary Fe values of OSF group $(49.28 \pm 3.25 \mu \mathrm{g} / \mathrm{dl})$ too reduced on comparison with controls $(59.34 \pm 4.50 \mu \mathrm{g} / \mathrm{dl})$.

Conclusions: It is important that doctors and other health professionals are aware of the nutritional essentiality of micronutrients, and for the situations where an increased intake may lead to clinical benefit. The present study highlighted the importance of micronutrients in oral health and their role in diagnosis or prognostic estimation of OSF.

Key words: Micronutrients; Salivary analysis; Oral sub mucous fibrosis; Oral potentially malignant disorder

\section{Introduction}

There is growing interest in the role of the micronutrients (essential trace elements and vitamins) in optimising health, and in prevention or treatment of disease. It is important that doctors and other health professionals are aware of the evidence for the nutritional essentiality of micronutrients, and for the situations where an increased intake may lead to clinical benefit. People from poorer background may well take less fresh fruit and vegetables.
Such groups may benefit from some form of micronutrient supplement, although direct comparisons are lacking of provision of supplements rather than improving dietary intake [1]. A large body of evidence suggests that a significant percentage of deaths resulting from cancer could be avoided through greater attention to proper and adequate nutrition. There are many studies in the literature, assessing role of micronutrients in oral cancer and pre cancer, which are carried through serum analysis. However, 
there are not many studies assessing salivary micronutrients in oral precancerous diseases like Oral Sub Mucous Fibrosis (OSF), which is a chronic, progressive, scarring condition characterized by oral mucosal blanching and rigidity Figure 1. It has been associated with long-lasting dipping of betel-nut or paan in the mouth $[2,3]$.

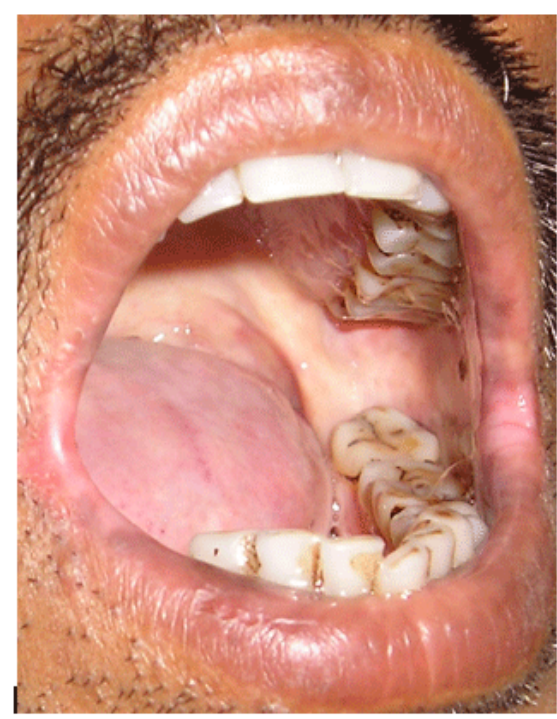

Figure 1: Oral mucosal blanching and paan stains over the teeth in a patient with OSF

Like blood, saliva is a complex fluid containing a variety of enzymes, hormones, antibodies, antimicrobial constituents, and growth factors. Considering the fact that sound nutritional habits and a sufficient supply of essential vitamins and minerals are of considerable importance for oral health we aimed to evaluate whether salivary micronutrients play any role in diagnosis or prognostic estimation in untreated cases of oral precancerous conditions like OSF [4].

\section{Materials and Methods}

A total of 84 subjects ranging in age from 21 to 65 years, were included in the prospective study that was approved by the ethical committee of the institute. After obtaining informed written consent from the subjects participated in the study, a thorough habit history including duration and frequency of areca nut or paan chewing habits was obtained. The subjects were divided into two groups. The first group consisted of 40 clinically diagnosed and histopathologically confirmed cases of OSF. The second group comprised 44 age and sex matched healthy controls without any history of areca nut or paan abuse and devoid of any evident oral lesions. Subjects with previous history of malignancy, long-term drug intake, systemic illness or history of antioxidant supplement were excluded from the study.

\section{Analysis of Salivary Micronutrients}

The subjects were requested not to eat, drink, perform oral hygiene activities or chew 60 minutes prior to the saliva collection procedure. The participants were then seated on the dental chair, asked to rinse the mouth and instructed to spit in a beaker every one minute till $5 \mathrm{ml}$ of saliva was obtained. The saliva was then transferred to plastic tubes and coded accordingly to avoid any bias during laboratory analysis. These salivary samples were centrifuged at $2500 \mathrm{rpm}$ for 10 minutes. The supernatant was collected and stored at $-20^{\circ} \mathrm{C}$ until further analysis. The saliva was then analysed for micronutrients. Zinc and $\mathrm{Cu}$ level were analysed using atomic absorption spectrometer and Fe using spectrophotometer.

\section{Statistical Analysis}

The results obtained after tabulation were analysed statistically using Statistical Package for the Social Sciences (SPSS) version 18.0 for Windows. The data were expressed as mean values \pm standard deviation. The mean values in the groups were compared with one way analysis of variance (ANOVA) followed by a post hoc analysis using Least Significant Difference (LSD) test. The P values of less than 0.05 were considered to show statistical significance.

\section{Results}

In the present study, mean age of patients with OSF was $38.28 \pm 2.23$ years. Among them, $31(77.5 \%)$ were males and $9(22.5 \%)$ were females. In our study, the mean salivary $\mathrm{Cu}$ values of OSF group $(127.30 \pm 6.77 \mu \mathrm{g} / \mathrm{dl})$ has increased compared to that of controls $(112.68 \pm 7.95 \mu \mathrm{g} / \mathrm{dl})$ but it was statistically non-significant. The mean salivary $\mathrm{Zn}$ values of OSF group $(116.80 \pm 9.93 \mu \mathrm{g} / \mathrm{dl})$ was significantly decreased $(P<0.01)$ compared to that of controls $(146.76 \pm 9.17 \mu \mathrm{g} / \mathrm{dl})$. The mean salivary Fe values of OSF group $(49.28 \pm 3.25 \mu \mathrm{g} / \mathrm{dl})$ too reduced on comparison with controls $(59.34 \pm 4.50 \mu \mathrm{g} / \mathrm{dl})$, but the results were statistically non-significant Table 1 .

\begin{tabular}{|c|c|c|c|}
\hline \multicolumn{4}{|c|}{ Table 1: Determination of salivary micronutrients } \\
\hline Subjects & Copper $(\boldsymbol{\mu g} / \mathbf{d l})$ & Zinc $(\boldsymbol{\mu g} / \mathbf{d} \mathbf{l})$ & Iron $(\boldsymbol{\mu g} / \mathbf{d l})$ \\
\hline $\begin{array}{c}\text { Controls } \\
(\mathrm{n}=44)\end{array}$ & $112.68 \pm 7.95$ & $146.76 \pm 9.17$ & $59.34 \pm 4.50$ \\
\hline OSF (n=40) & $127.30 \pm 6.77$ & $116.80 \pm 9.93 \mathrm{a}^{*}$ & $49.28 \pm 3.25$ \\
\hline \multicolumn{3}{|l|}{$\begin{array}{l}\text { Values are expressed as mean } \pm \text { S.D } \\
\text { a - OSF compared to controls; }{ }^{*} P<0.01\end{array}$} \\
\hline
\end{tabular}

\section{Discussion}

Many dietary compounds have been considered to contribute in cancer prevention, which plays a pivotal role in host defence against the initiation and promotion of several malignancies. Bioelements like $\mathrm{Cu}$ is involved in vital biochemical activities like different redox and free radical formation and in maintaining cellular proton homeostasis [5]. Copper is involved in the cell metabolism, and is a part of various enzymes such as tyrosinase, uricase, and cytochrome oxidase, which are mainly concerned with oxidation reaction. Copper is available in the liver, shellfish, dried fruit, milk and milk products, sunflower seeds, oysters, sesame seeds, tahini, and sun-dried tomatoes. High copper intake for prolonged period causes increased copper percentages in 
serum and tissue that in turn causes oxidative stress and affects several immune functions. Decreased copper levels are observed in few malignancies, mostly in the tumours which have high catabolic rate or which is of highly metastatic type [6]. Copper has been the most extensively studied of the trace elements in patients with malignant diseases and these elements in serum has been found to be reliable parameter as a diagnostic and prognostic index in case of craniofacial tumours. Our study showed increase in salivary $\mathrm{Cu}$ in OSF group compared to controls Table 1 which could be due to sequestration of $\mathrm{Cu}$ from pre-cancerous tissue to oral cavity, which is bathed by saliva. Further, the patients with OSF had the habit of chewing areca-nut or paan. Copper, being one of the main ingredients of betel-nut and paan could have diffused into saliva showing higher values. Area plant growers periodically spray copper sulphate on to the areca nut to prevent fungal infection of the areca nut. This adds to higher copper content of areca nut. On chewing areca nut, copper diffuses into the oral mucosa. Chewing areca nut for 5-30 minutes increases the soluble copper levels in the oral fluids [7].

Zinc, an essential trace element for the growth of humans and other animals, has a unique and extensive role in biological processes [8]. Zinc is essential for regulation of cell cycle and cell division. It is also essential for DNA polymerase activity and is particularly important for rapid cell proliferation encountered in growing tumours. In addition, it has been reported that $\mathrm{Zn}$ deficiency might produce marked effects on about all components of the immune system [9]. Zinc is one of the antioxidants in food on which the activities of enzymes of the antioxidant system of the body, such as superoxide dismutase, depend. Moreover, Zn may directly prevent DNA damage and eventually gene mutation, and in this way, the element decreases the risk of cancer accordingly. It was suggested that $\mathrm{Zn}$ deficiency is a potential risk factor for oral and periodontal diseases [10]. We analysed salivary $\mathrm{Zn}$ in our patients OSF to investigate its possible role in pre-cancer development and progression. We found that salivary $\mathrm{Zn}$ values were significantly lower in pre-cancerous patients of our study samples which histologically showed dysplasia. In another study, serum levels $\mathrm{Zn}$ in oral cancer patients were significantly higher than in control group [11]. The dysplastic cells and tissues have increased metabolic requirement of Zn, which results in an increased uptake from adjacent structure such as salivary secretion. This could be the reason for decreased Zn levels in OSF salivary samples in our study. Most of our patients with OSF had poor financial and educational background, which resulted in decreased intake of nutritional diet. This also could have contributed to $\mathrm{Zn}$ deficiency in our patients. Chewing of areca nut or paan is reported to supress hunger, which may lead to decreased intake of nutritional diet.

Iron is an important micronutrient, which is utilized, in human body for the transportation of oxygen, haematopoiesis, production of enzymes, and immune surveillance. Body $\mathrm{Fe}$ absorption is controlled by the duodenal mucosa, which allows the intake of appropriate quantities of iron to balance, exactly, small daily losses [12]. Iron is an important micronutrient that ensures the development of normal red blood cells and healthy immune function. Iron deficiency is the most common and widespread nutritional deficiency in the world - affecting the very poor, especially women - and is the cause of about half of all cases of anaemia. An association between oral pre-malignancy and low iron intake was found in Indian women, but it was statistically non-significant [13]. In experimental studies of 4-nitroquinoline$\mathrm{N}$-oxide (4-NQO) exposed laboratory rats, the incidence of oral tumours was significantly elevated in iron deficient animals although these differences were not confirmed in subsequent studies [14]. The mechanism by which iron deficiency may induce oral cancer may involve the induction of oxidative stress through the production of free radicals and reactive oxygen species that are potentially able to induce cellular injury.

The serum Fe level of patients with OSF was correlated with histopathological grading of OSF. The results of the study showed that serum levels consistently decreased with the progression of histopathological grading of OSF [15]. We have considered salivary Fe estimation to observe whether we get similar results as serum estimation. Our study too showed decrease in salivary Fe values of OSF patients compared to controls as the disease progressed Table 1. As a micronutrient, body Fe would have been utilized for progression of OSF. Iron may also have been used for the excessive collagen synthesis occurring during progression of OSF. Further, most of our patients had poor oral hygiene habits; leading to loss of teeth supporting structures, called periodontitis. Gingivitis, which is the inflammation of gingiva is the precursor of periodontitis. Gingivitis and periodontitis both lead to bleeding of gums. This continued minor amount of bleeding in the oral cavity of our patients might have also led to iron deficiency. These findings alert health care providers (especially dentists) to educate the patients with regards to oral care and maintenance of healthy periodontium by proper brushing habits and regular visits to dentists for oral prophylaxis. These measures along with nutritional diet may improve the iron content in the body. There was significant negative relationship between age and salivary Fe content in control group of our study. It was observed that as age advanced, the dental patients' oral health got deteriorated including loss of teeth. Very few patients reported for teeth replacement once they had lost their teeth. The lack of teeth in the oral cavity led to decreased chewing efficiency resulting in decreased nutritious food intake. This could be the reason for decreased salivary Fe in control group of our study. This finding demands dental health education to public, emphasizing replacement of missing teeth by artificial denture in order to facilitate effective chewing of nutritious food.

We conclude that doctors and other health professionals are aware of the nutritional essentiality of micronutrients, and for the situations where an increased intake may lead to clinical benefit. The present study highlighted the importance of micronutrients in oral health and their role in diagnosis or prognostic estimation of OSF. Further research in estimating the role of micronutrients in various oral diseases may reveal interesting data in optimising health, prevention or treatment of oral diseases. 


\section{References}

1. Hoare J, Henderson L, Bates CJ. The national diet and nutrition survey: adults aged 19-64. The Stationery Office, London. 2004.

2. George A, Varghese SS, Thomas J, Gopakumar D, Mani V. Potentially Malignant disorders of oral cavity. OMPJ. 2011;2:95-100.

3. Angadi PV, Rekha KP. Oral submucous fibrosis: a clinico-pathologic review of 205 cases in Indians. Oral Maxillofac Surg. 2011;15(1):1519. doi: $10.1007 / \mathrm{s} 10006-010-0225-\mathrm{x}$

4. Willershausen B, Ross A, Försch M, Willershausen, Mohaupt PH, Callaway A. The Influence of Micronutrients on Oral and General Health. Eur J Med Res. 2011;16(11):514-518. doi: 10.1186/2047783X-16-11-514

5. Paul RR, Chaterjee J, Das AK, Cervera ML, DeLa Guardia M, Chaudhur $\mathrm{K}$. Altered elemental profile as indicator of homeostatic imbalance in pathogenesis of oral submucous fibrosis. Biol Trace Elem Res. 2002;87(1-3):45-56. doi: 10.1385/BTER:87:1-3:045

6. Prashanth L, Kattapagari KK, Chitturi RT, Venkat Ramana Reddy Baddam, Lingamaneni Krishna Prasad. A review on role of essential trace elements in health and disease. Journal of Dr. NTR University of Health Sciences. 2015;4(2):75-85.

7. Prabhu RV, Prabhu V, Chatra L Shenai P, Prashant Shenai, Nithin Suvarna and Savita Dandekeri. Areca nut and its role in oral submucous fibrosis. J Clin Exp Dent. 2014;6(5):e569-e575. doi: 10.4317/jced.51318
8. Ackland ML, Michalczyk A. Zinc deficiency and its inherited disorders-a review. Genes Nutr. 2006;1(1):41-49. doi: 10.1007/BF02829935

9. Seyedmajidi SA, Seyedmajidi M, Moghadamnia A, Khani Z, Zahedpasha $S$ Jenabian N. Effect of Zinc-Deficient Diet on Oral Tissues and Periodontal Indices in Rats. Int J Mol Cell Med. 2014;3(2):81-87.

10. Orbak R, Kara C, Ozbek E, Tezel A, Demir T. Effects of zinc deficiency on oral and periodontal diseases in rats. J Periodontal Res. 2007;42(2):138-143.

11. Baharvand M, Manifar S, Akkafan R, Mortazavi H, Sabour S. Serum Levels of Ferritin, Copper, and Zinc in Patients with Oral Cancer. Biomed J. 2014;37(5):331-336. doi: 10.4103/2319-4170.132888

12. Rennie JS, MacDonald DG, Dagg JH. Iron and the oral epithelium: a review. J R Soc Med. 1984;77(7):602-607. doi: $10.1177 / 014107688407700714$

13. Gupta PC, Hebert JR, Bhonsle RB, Murti PR, Mehta H, Mehta FS. Influence of dietary factors on oral precancerous lesions in a population-based case-control study in Kerala, India. Cancer. 1999;85(9):1885-1893.

14. Prime SS, MacDonald DG, Rennie JS. The effect of iron deficiency on experimental oral carcinogenesis in the rat. $\mathrm{Br} \mathrm{J}$ Cancer. 1983;47(3):413-418.

15. Shetty SR, Babu S, Kumari S, Shetty P, Vijay R, Karikal A. Evaluation of micronutrient status in serum and saliva of oral sub mucous fibrosis patients: A clinicopathological study. Indian J Med Paediatr Oncol. 2012;33(4):224-226. doi: 10.4103/0971-5851.107087 